\title{
Non-extractable proanthocyanidins from grapes are a source of bioavailable (epi)catechin and derived metabolites in rats
}

\author{
María Luisa Mateos-Martín ${ }^{1}$, Jara Pérez-Jiménez ${ }^{1 *}$, Elisabet Fuguet ${ }^{1,2}$ and Josep Lluís Torres ${ }^{1}$ \\ ${ }^{1}$ Institute for Advanced Chemistry of Catalonia (IQAC-CSIC), Jordi Girona 18-26, O8034 Barcelona, Spain \\ ${ }^{2}$ Departament de Química Analítica, Universitat de Barcelona, Martí i Franquès 1-11, O8028 Barcelona, Spain \\ (Submitted 10 June 2011 - Final revision received 14 September 2011 - Accepted 19 September 2011 - First published online 6 December 2011)
}

\begin{abstract}
The non-extractable fraction of many fruit and vegetables contains putatively bioactive polyphenolic compounds that, in most cases, have not been well characterised structurally. Non-extractable proanthocyanidins (NEPA) of a polymeric nature are part of the dietary fibre fraction of food. Using liquid chromatography coupled to a mass spectrometer equipped with an electrospray ionisation chamber and a triple quadrupole mass analyser for tandem analysis (HPLC-ESI-QqQ-MS/MS) techniques, we examine the phenolic metabolites present in urine and faeces from rats $24 \mathrm{~h}$ after ingestion of an NEPA-rich fraction. We show that NEPA are partially depolymerised during their transit along the intestinal tract, as evidenced by the presence of (epi)catechin (EC) monomers and dimers in faeces and phase II conjugates of EC in urine. Moreover, NEPA are further metabolised by the intestinal microbiota into smaller metabolites including phenolic acids that are present in urine as both free phenolics and conjugates with glucuronate or sulphate moieties. For the first time, we report evidence that NEPA behave in vivo as a source of phenolics that are released progressively and deliver phenolic species that come into contact with the intestinal walls and are bioavailable for at least $24 \mathrm{~h}$ after ingestion.
\end{abstract}

Key words: Bioavailability: MS: Non-extractable proanthocyanidins: Polyphenols

Proanthocyanidins (PA) are a class of dietary polyphenols. They are polymers of flavan-3-ols present in a wide variety of plant-based foodstuffs, such as berries, cocoa or certain nuts $^{(1)}$. Several supplementation studies both in animals and in human subjects using PA-rich products have shown that PA play a preventive role against several conditions including $\mathrm{CVD}^{(2-4)}$ or diabetes ${ }^{(1,5,6)}$. Similarly, a recent epidemiological study showed an inverse association between the intake of polymeric PA and the risk of colorectal cancer ${ }^{(7)}$. To advance knowledge of the possible effects of PA on human health, it is important to characterise their metabolic fate in detail, that is to say, the degradation they may suffer once ingested and the distribution of their metabolites through the different organs and fluids.

Most studies of PA, including those that address their metabolism, assume that the PA in foodstuffs correspond exclusively to the supernatants obtained after extracting the food with acetone; the most common procedure for their analysis ${ }^{(8)}$. However, these PA would actually only correspond to the extractable proanthocyanidins (EPA), which represent only a fraction of dietary PA. Recent work has emphasised that a considerable proportion of $\mathrm{PA}$, the non-extractable proanthocyanidins (NEPA), remains in the residue from such extractions $^{(9)}$ and may very well play a more significant functional role than EPA. NEPA are associated with other components of the food matrix, mainly dietary fibre, and in fact constitute a part of it, according to current definitions of dietary fibre ${ }^{(10,11)}$. To date, there is no common method for determining NEPA. They are usually determined by destructive spectrophotometric methods and only a few recent papers have attempted structurally meaningful analysis ${ }^{(12,13)}$; therefore, structural evidence regarding the composition of NEPA as essential constituents of many foodstuffs is still scarce. The currently available evidence suggests that NEPA may be more abundant than EPA in much food ${ }^{(12-14)}$ and, therefore, that significant amounts of NEPA are ingested daily.

Over the last decade, several studies have addressed the metabolism of PA. Although initial studies emphasised fairly poor intestinal absorption that was limited to dimers ${ }^{(15-17)}$, later observations indicate that once intact $\mathrm{PA}$ reach the colon they are widely transformed by the colonic microbiota into small phenolic acids ${ }^{(18-21)}$. These metabolites are absorbed, and then transformed in the liver, and the resulting conjugates are transferred to the bloodstream. A recent study

Abbreviations: EC, (epi)catechin; EPA, extractable proanthocyanidins; GADF, grape antioxidant dietary fibre; GlcA, glucuronide; MRM, multiple reaction monitoring; NEPA, non-extractable proanthocyanidins; PA, proanthocyanidins; ppm, parts per million. 
in which $\left[{ }^{14} \mathrm{C}\right]$ procyanidin $\mathrm{B} 2$, a labelled PA dimer, was administered to rats, reported bioavailability of around $80 \%$, based on total urinary ${ }^{14} \mathrm{C}^{(20)}$.

Nevertheless, these papers have mostly addressed the bioavailability of dimers or trimers, while the most abundant PA in food are polymers ${ }^{(22)}$. Indeed, some recent studies have suggested that PA are also depolymerised into (epi)catechin (EC) units before cleavage into smaller species and further metabolism ${ }^{(21,23-25)}$. These studies suggest that phenolics that are bioavailable after the ingestion of PA-rich foodstuffs must have come from $\mathrm{NEPA}^{(24-26)}$, but this has not been proved as the specific metabolism of NEPA has never been reported.

Grape antioxidant dietary fibre (GADF) is a food product obtained from red grapes that is rich in dietary fibre and polyphenols ${ }^{(27)}$. Besides extractable polyphenols, including $\mathrm{EPA}^{(28)}$, GADF contains a significant amount (14.8\%) of $\mathrm{NEPA}^{(27)}$ and was used in the studies which suggest that non-extractable polyphenols are an important source of metabolites that are bioavailable in rats ${ }^{(24-26)}$. To study the contribution of NEPA to the pool of phenolic metabolites from fruit and vegetables, we considered using a NEPA-rich fraction from GADF.

Our objective was to evaluate the fate of NEPA in rats $24 \mathrm{~h}$ after ingestion of a preparation free from any extractable polyphenols. NEPA metabolites, including hepatic and microbially derived metabolites, were analysed in urine and faeces using liquid chromatography coupled to a mass spectrometer equipped with an electrospray ionisation (ESI) chamber and a triple quadrupole mass analyser for tandem analysis (HPLC-ESI-QqQ-MS/MS).

\section{Experimental methods}

\section{Reagents and samples}

GADF was obtained from red grapes (Cencibel variety, harvested in 2005 in La Mancha region of Spain) by a patented procedure $^{(29)}$. The NEPA content of GADF has previously been reported to be $14.8 \mathrm{~g} / 100 \mathrm{~g}$ of dry weight ${ }^{(27)}$. To obtain an EPA-free (and therefore NEPA-rich) fraction, GADF ( $4 \mathrm{~g}$ ) was defatted with hexane $(3 \times 40 \mathrm{ml})$, air-dried overnight and the residue was extracted with methanol-water (50:50, $\mathrm{v} / \mathrm{v}, 200 \mathrm{ml}$ ) and then with acetone-water-acetic acid (70:29.5:0 5 , by vol., $200 \mathrm{ml}$ ) once each at room temperature. The supernatant was decanted and the residue, including the NEPA-rich fraction, was vacuum filtered and lyophilised. The actual NEPA content of this residue is around $25 \%$ according to published information ${ }^{(26)}$.

Standards of EC ( $\geq 97 \%), 3-$ and 4-hydroxyphenylacetic acid $(\geq 98 \%)$, 3,4-dihydroxyphenylacetic acid $(\geq 98 \%)$, 3- and 4-hydroxybenzoic acid ( $\geq 97 \%$ ), vanillic acid ( $\geq 97 \%)$, caffeic acid ( $\geq 95 \%)$, 3,4-di-hydroxyphenylpropionic acid ( $\geq 98 \%$ ), 4-hydroxyphenylpropionic acid ( $>98 \%$ ), protocatechuic acid $(\geq 97 \%)$, ferulic acid $(\geq 99 \%)$, isoferulic acid $(\geq 97 \%), \quad p$-coumaric acid $(\geq 98 \%), \quad m$-coumaric acid $(\geq 97 \%)$ and taxifolin $(\geq 85 \%)$ were obtained from Sigma Chemical (St Louis, MO, USA). Methanol (analytical grade), phosphoric acid $(\geq 85 \%)$ and acetic acid were purchased from Panreac (Castellar del Vallès, Barcelona, Spain). Acetonitrile (HPLC grade) and formic acid (analytical grade) were obtained from Merck (Darmstadt, Germany). Water was purified by a Milli-Q plus system from Millipore (Bedford, MA, USA) to a resistivity of $18.2 \mathrm{~m} \Omega / \mathrm{cm}$.

\section{Animal experiments}

The study was carried out on female Sprague-Dawley rats ( $n$ 10, body weight 233 (sD 9.3) g, 12 weeks of age) provided by Harlan Interfauna Ibérica SL (Barcelona, Spain). The animals were fed with a polyphenol-free diet (TD94048), also purchased from Harlan Interfauna Ibérica SL, and they were maintained in plastic cages at room temperature $\left(22 \pm 2^{\circ} \mathrm{C}\right.$ ) and 55 (SD 10)\% relative humidity, with a $12 \mathrm{~h}$ light $-12 \mathrm{~h}$ dark cycle for 1 week, in accordance with European Union regulations. After food deprivation for $12 \mathrm{~h}$ with free access to water, a group of animals ( $n$ 5) was administered a suspension of NEPA from GADF in tap water $(1 \mathrm{~g}$ NEPA-rich fraction/10 ml, $1.6 \mathrm{~g}$ NEPA-rich fraction/kg body weight) by oral gavage, while a control group ( $n$ 5) was administered tap water $(16 \mathrm{ml} / \mathrm{kg}$ body weight). The animals were then placed in metabolism cages and urine and faeces were collected over $24 \mathrm{~h}$ and stored at $-80^{\circ} \mathrm{C}$ until extraction and analysis. These experimental protocols were approved by the Experimental Animal Ethical Research Committee of the CSIC in accordance with the current regulations for the use and handling of experimental animals.

\section{Sample preparation}

The biological samples were prepared according to previously described procedures for the extraction of phenolic metabolites ${ }^{(21,24,25)}$. Briefly, urine samples were concentrated via a nitrogen stream at room temperature and then resuspended in $1 \mathrm{ml}$ of acid water (addition of phosphoric acid to reach $\mathrm{pH} 3)$. Taxifolin $(100 \mu$ l of a 50 parts per million (ppm) solution) was added as an internal standard, to obtain a final concentration of $5 \mathrm{ppm}$. Then the samples were subjected to solid phase extraction in Oasis HLB (60 mg) cartridges from Waters Corporation (Mildford, MA, USA). The cartridges were activated with methanol $(1 \mathrm{ml})$ and acid water $(2 \mathrm{ml})$ and the samples loaded. To remove interfering components, the samples were washed with acid water $(9 \mathrm{ml})$ and then the phenolic compounds were eluted with methanol $(1 \mathrm{ml})$.

Faeces $(0.5 \mathrm{~g})$ were defatted with hexane $(10 \mathrm{ml})$ and the residue was extracted with methanol-water-phosphoric acid (8:199:0.1, by vol., $10 \mathrm{ml})$ and concentrated down to $1 \mathrm{ml}$ by nitrogen stream at room temperature. Taxifolin (100 $\mu$ l of a $50 \mathrm{ppm}$ solution, final concentration $5 \mathrm{ppm}$ ) was added to each sample as an internal standard.

Extracts from both urine and faeces were filtered through a polytetrafluoroethylene $0 \cdot 45-\mu \mathrm{m}$ membrane from Waters Corporation into amber vials for HPLC-MS/MS analysis. 


\section{HPLC-electrospray ionisation-MS/MS analysis}

A Quatro LC from Waters Corporation triple quadrupole mass spectrometer with an electrospray source was used in negative mode to obtain MS and MS/MS data. Liquid chromatography separations were performed using an Alliance 2695 system from Waters Corporation equipped with a Phenomenex (Torrance, CA, USA) Luna C18 $(50 \times 2 \cdot 1 \mathrm{~mm}$ internal diameter) $3.5 \mu \mathrm{m}$ particle size column and a Phenomenex Securityguard C18 $(4 \times 3 \mathrm{~mm}$ internal diameter $)$ column. Gradient elution was performed with a binary system consisting of (A) $0 \cdot 1 \%$ aqueous formic acid and (B) $0.1 \%$ formic acid in $\mathrm{CH}_{3} \mathrm{CN}$. An increasing linear gradient $(\mathrm{v} / \mathrm{v})$ was used ( $t$ (min), \%B): 0, 8; 10, 23; 15, 50; 20, 50; 21, 100, followed by a re-equilibration step.

Metabolites were detected in multiple reaction monitoring (MRM) experiments, and their identity was confirmed by product ion scan experiments. The dwell time for the MRM experiments was $100 \mathrm{~ms}$ and the cycle time for all the experiments was $2 \mathrm{~s}$. Cone energy and collision energy in MRM mode were optimised for each group of metabolites: $30 \mathrm{~V}$ and $10 \mathrm{eV}$ for taxifolin, $30 \mathrm{~V}$ and $15 \mathrm{eV}$ for microbially derived phenolic metabolite derivatives, $30 \mathrm{~V}$ and $20 \mathrm{eV}$ for $\mathrm{EC}$, and $40 \mathrm{~V}$ and $20 \mathrm{eV}$ for $\mathrm{EC}$ derivatives.

\section{Results}

\section{(Epi)catechin and its phase II metabolites}

Free EC (MRM transition $289 \rightarrow 245$ ) and a signal corresponding to a dimer $(577 \rightarrow 289)$ were detected in faeces by liquid chromatography-ESI-MS/MS. The product ion spectrum of this dimer provided characteristic fragments at $\mathrm{m} / z 425$ and $m / z 405$ originated by cleavage of the C-ring of one of the EC units through a retro Diels-Alder reaction and consecutive loss of water. A fragment at $m / z$ 451, caused by heterolytic ring fission, was also observed.

A total of ten EC conjugates derived from the activity of phase-II enzymes in both the intestinal tract and liver were detected in urine samples (Table 1). All these compounds were either not detected in the control group or detected at concentrations that were at least 10 -fold lower. The metabolites were initially identified by previously reported MRM transitions corresponding to the main fragments ${ }^{(24,30)}$ and their identity was confirmed by a second MRM transition and/or by product ion scan experiments.

The EC metabolites included three glucuronidated forms ( $465 \rightarrow 289$ ), two sulphated forms $(369 \rightarrow 289)$, a monoconjugated metabolite with glutathione $(594 \rightarrow 289)$, two methylated and glucuronidated forms $(479 \rightarrow 303)$, a di-glucuronidated form $(641 \rightarrow 289)$ and a tri-conjugated metabolite $(397 \rightarrow 289)$ corroborated by MS/MS experiments. All these derivatives were detected in urine samples and the di-glucuronidated conjugate was also detected in faeces.

Fig. 1 shows the product ion spectrum of glucuronide (GlcA)EC-3 $(m / z 465)$. Fragments at $m / z 289$ and $m / z 245$, corresponding to the loss of the conjugate moiety and the respective cleavage of $\mathrm{CO}_{2}$ from the free EC unit, were observed; as were two fragments at $m / z 175$ and 113 , from the fragmentation of the GlcA moiety. The MS/MS spectra of other conjugates showed characteristic fragments corresponding to a B-ring retro Diels-Alder fission of the EC, such as the fragment at $\mathrm{m} / \mathrm{z}$ 137 for Me-GlcA-EC, which corresponds to a B-ring fragment with attached methyl group and GlcA moieties, indicating that the conjugation was located on the B-ring.

\section{Microbially derived proanthocyanidin metabolites}

A total of twenty microbially derived PA metabolites were identified in urine from rats fed the NEPA-rich fraction; two of them were also detected in faeces (Table 2). All these metabolites were either not detected in the control group or detected at concentrations that were at least 10-fold lower. Microbially derived PA metabolites detected in urine included valerolactones, phenylvaleric acids, phenylpropionic acids, phenylacetic acids, benzoic acid, cinnamic acids and lignans. 3,4-Dihydroxyphenylacetic acid, detected by MRM transition $(167 \rightarrow 123)$, has been reported to be as a specific metabolite of polymeric PA, since it has never been identified in studies performed with pure monomeric flavan-3-ols ${ }^{(31)}$. 3,4-Dihydroxyphenylacetic acid may be metabolised to

Table 1. (-)-(Epi)catechin (EC) and conjugated metabolites in urine and faeces from rats fed a non-extractable proanthocyanidin-rich fraction from grape antioxidant dietary fibre

\begin{tabular}{|c|c|c|c|c|}
\hline Metabolite & MRM parent & Identification & Urine & Faeces \\
\hline$(-)-E C$ & $289 \rightarrow 245$ & Standard retention time & & $x$ \\
\hline PC dimer & $577 \rightarrow 289$ & MS/MS: 575; 451; 424; 405; 327; 289; 123 & & $x$ \\
\hline \multicolumn{5}{|c|}{ Mono-conjugated metabolites } \\
\hline GlcA-EC-1 & $465 \rightarrow 289$ & MS/MS: 465; 289; 245; 137; 113 & $x$ & \\
\hline GlcA-EC-2 & $465 \rightarrow 289$ & MS/MS: 465; $289 ; 257 ; 175 ; 113$ & $x$ & \\
\hline GlcA-EC-3 & $465 \rightarrow 289$ & MS/MS: 465; 289; 245; 175; 113 & $x$ & \\
\hline Sulf-EC-1 & $369 \rightarrow 289$ & MS/MS: 369; 289; 245; 228; 184; 113 & $x$ & \\
\hline Sulf-EC-2 & $369 \rightarrow 289$ & MRM daughter $289 \rightarrow 245$ & $x$ & \\
\hline GSH-EC-1 & $594 \rightarrow 289$ & MRM daughter $289 \rightarrow 245$ & $x$ & \\
\hline \multicolumn{5}{|c|}{ Di-conjugated metabolites } \\
\hline Me-GIcA-EC-1 & $479 \rightarrow 303$ & MS/MS: 479; 303; 289; 245; 173; 137; 113 & $x$ & \\
\hline Me-GlcA-EC-2 & $479 \rightarrow 303$ & MS/MS: 479; 303; 285; 259; 173; 137; 113 & $x$ & \\
\hline Di-GlcA-EC-1 & $641 \rightarrow 289$ & MS/MS: 641; 465; 289 & $x$ & $x$ \\
\hline \multicolumn{5}{|c|}{ Tri-conjugated metabolites } \\
\hline Di-Me-Sulf-EC-1 & $397 \rightarrow 289$ & MRM daughter $289 \rightarrow 245$ & $x$ & \\
\hline
\end{tabular}

MRM, multiple reaction monitoring; PC, procyanidins; GlcA, glucuronide; Sulf, sulphate; GSH, glutathione; Me: methyl group. 


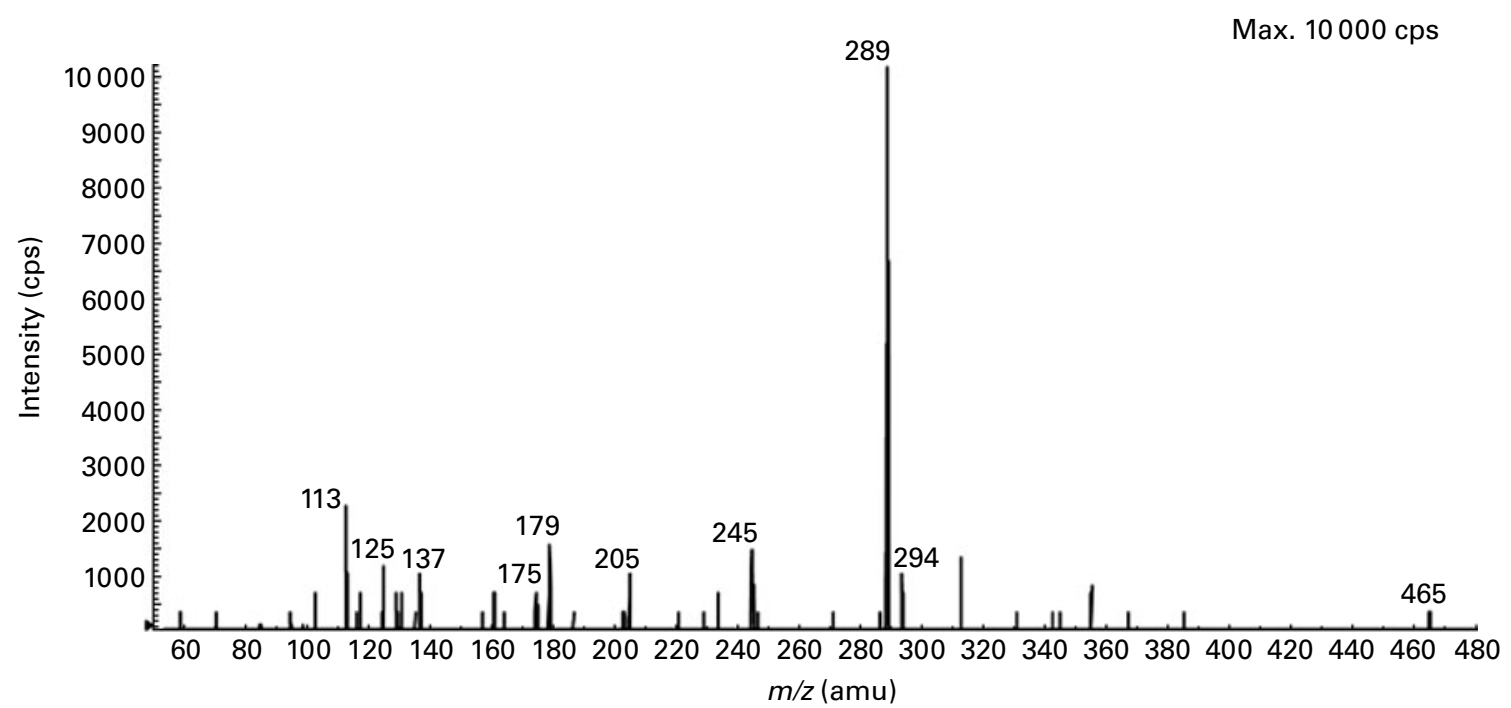

Fig. 1. HPLC-electrospray ionization-MS/MS product ion scan spectrum of glucuronide-(epi)catechin ( $m / z$ 465). cps, Counts per second; amu, atomic mass unit; max., maximum.

3-hydroxyphenylacetic acid and 4-hydroxyphenylacetic acid $(151 \rightarrow 107)$, which were also detected. Similarly, 3,4-dihydroxyphenylpropionic acid, 3-hydroxyphenylpropionic acid and 4-hydroxyphenylpropionic acid were detected in urine. Both 3,4-dihydroxyphenylacetic acid and 3,4-dihydroxyphenylpropionic acid were absorbed and later conjugated in the liver as shown by the detection of the derivatives GlcA-3 or 4-hydroxyphenylacetic acid (327 $\rightarrow 151)$, Sulf-3, 4-dihydroxyphenylpropionic acid $(261 \rightarrow 181)$ and Sulf3 or 4 -hydroxyphenylpropionic acid $(245 \rightarrow 165)$. Conjugated forms of phenylvaleric and hippuric acid were detected in urine.

Two microbially derived phenolic metabolites were identified in the faeces from rats fed the NEPA-rich fraction: 4-hydroxyphenylpropionic acid $(165 \rightarrow 121)$ and 3,4-dihydroxyphenylpropionic acid $(181 \rightarrow 137)$.

Phenolic acids generated fragments corresponding to the successive loss of two $\mathrm{CO}_{2}$ molecules. Similarly, MS/MS spectra of sulphated forms showed signals corresponding to the loss of sulphate and $\mathrm{CO}_{2}$. These fragments confirm the identity

Table 2. Detection of microbially derived proanthocyanidin metabolites in urine and faeces from rats fed a nonextractable proanthocyanidin-rich fraction from grape antioxidant dietary fibre

\begin{tabular}{|c|c|c|c|c|}
\hline Metabolite & MRM parent & Identification & Urine & Faeces \\
\hline \multicolumn{5}{|l|}{ Valerolactones } \\
\hline Sulf-dihydroxyphenylvalerolactone & $287 \rightarrow 207$ & MRM daughter $207 \rightarrow 163$ & $x$ & \\
\hline \multicolumn{5}{|l|}{ Phenylvaleric acids } \\
\hline Sulf-dihydroxyphenylvaleric acid & $289 \rightarrow 209$ & MRM daughter $209 \rightarrow 165$ & $x$ & \\
\hline \multicolumn{5}{|l|}{ Phenylpropionic acids } \\
\hline 4-Hydroxyphenylpropionic acid & $165 \rightarrow 121$ & MS/MS: 165; 121; 93 & $x$ & $x$ \\
\hline 3,4-Dihydroxyphenylpropionic acid & $181 \rightarrow 137$ & Standard retention time & $x$ & $x$ \\
\hline Sulf-3,4-dihydroxyphenylpropionic acid & $261 \rightarrow 181$ & MRM daughter $181 \rightarrow 137$ & $x$ & \\
\hline Suf-3 or 4-hydroxyphenylpropionic acid & $245 \rightarrow 165$ & MS/MS: 245; 165; 121 & $x$ & \\
\hline \multicolumn{5}{|l|}{ Phenylacetic acids } \\
\hline 3-Hydroxyphenylacetic acid & $151 \rightarrow 107$ & Standard retention time & $x$ & \\
\hline 4-Hydroxyphenylacetic acid & $151 \rightarrow 107$ & Standard retention time & $x$ & \\
\hline GlcA-3- or 4-hydroxyphenylacetic acid & $327 \rightarrow 151$ & MS/MS: 327; 151; 107 & $x$ & \\
\hline 3,4-Dihydroxyphenylacetic acid & $167 \rightarrow 123$ & MS/MS: 167; 123; 105; 95 & $x$ & \\
\hline \multicolumn{5}{|l|}{ Benzoic acids } \\
\hline Benzoic acid & $121 \rightarrow 77$ & Standard retention time & $x$ & \\
\hline 4-Hydroxybenzoic acid & $137 \rightarrow 93$ & Standard retention time & $x$ & \\
\hline 3,4-Dihidroxybenzoic acid & $153 \rightarrow 109$ & Standard retention time & $x$ & \\
\hline Hippuric acid & $178 \rightarrow 134$ & Standard retention time & $x$ & \\
\hline Me-hippuric acid & $193 \rightarrow 178$ & MS/MS: 193; 178; 134 & $x$ & \\
\hline \multicolumn{5}{|l|}{ Cinnamic acids } \\
\hline$m$-Coumaric acid & $163 \rightarrow 119$ & Standard retention time & $x$ & \\
\hline$p$-Coumaric acid & $163 \rightarrow 119$ & Standard retention time & $x$ & \\
\hline Ferulic acid & $193 \rightarrow 134$ & Standard retention time & $x$ & \\
\hline \multicolumn{5}{|l|}{ Lignans } \\
\hline Enterodiol & $301 \rightarrow 107$ & Standard retention time & $x$ & \\
\hline Sulf-enterolactone & $377 \rightarrow 297$ & MRM daughter $297 \rightarrow 253$ & $x$ & \\
\hline
\end{tabular}

MRM, multiple reaction monitoring; Sulf, sulphate; GlcA, glucuronide; Me, methyl group. 
of some of the microbially derived metabolites; others were confirmed by the use of standards, i.e. 4-hydroxybenzoic acid (Fig. 2).

\section{Discussion}

Several studies have addressed the metabolism of dietary oligomeric PA, mostly dimers. That work provides quite a clear picture of the different steps in the metabolism of PA dimers in laboratory animals and human subjects ${ }^{(18-21)}$. This process comprises the absorption of monomers and small oligomers (dimers) of PA in the small intestine and the absorption of microbially derived metabolites in the large intestine, after direct fermentation of the oligomers by microbiota without prior depolymerisation into EC. The absorbed metabolites may be conjugated in the liver, mostly resulting in GlcA, sulphates and methyl derivatives which pass to the bloodstream and eventually reach other tissues. Finally, the metabolites are excreted in urine and the fraction of PA that is not absorbed is excreted in faeces.

The metabolic fate of larger PA polymers is believed to follow the same pattern as that of dimers and trimers: essentially, direct cleavage of the EC units into smaller phenolic acids by the intestinal microbiota. By examining the metabolic fate of GADF, we have recently suggested that polymeric PA undergo depolymerisation into EC units during their transit along the intestinal tract ${ }^{(24,25)}$. This is important because it implies that the polymers may gradually release EC moieties during the postprandial period. Using a NEPA-rich fraction, devoid of EC monomers and extractable oligomers, we show here that this is indeed occurring. The faeces of rats fed with NEPA contained monomeric and dimeric EC and
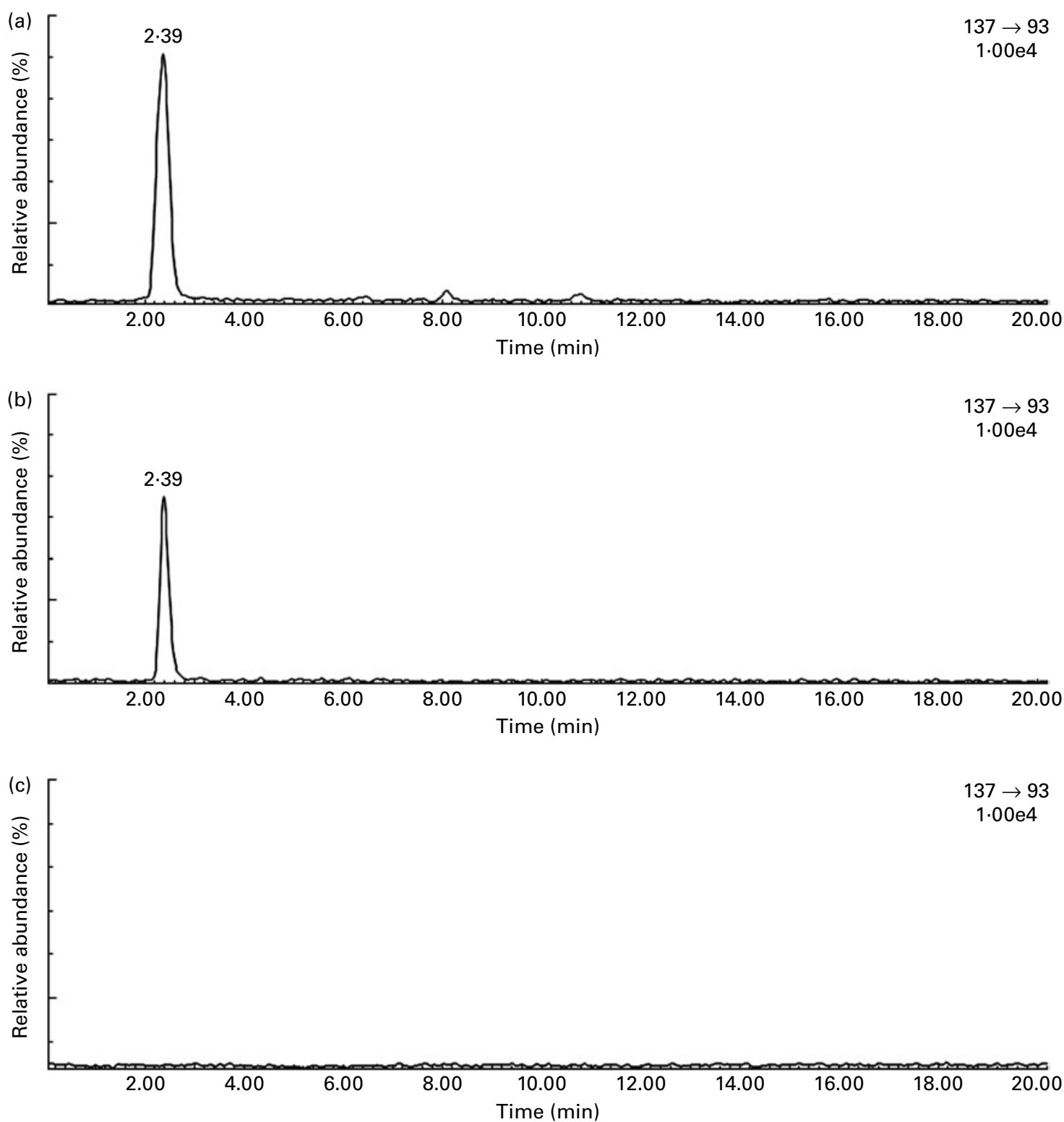

Fig. 2. HPLC-electrospray ionisation-MS profile corresponding to the detection by multiple reaction monitoring of 4-hydroxybenzoic acid (transition $137 \rightarrow 93$ ): (a) urine from rats fed non-extractable proanthocyanidins from grape antioxidant dietary fibre, (b) 4-hydroxybenzoic acid standard, (c) urine from rats fed water. 
their urine contained 10 phase II EC metabolites. These results clearly show that this fraction of dietary fibre generates bioavailable derivatives of EC. The di-glucuronidated EC derivative detected in faeces further demonstrates that those monomers that are released from NEPA efficiently enough to reach the liver suffer conjugation and are transported back to the intestine via bile. Our evidence for intestinal depolymerisation is consistent with a recent observation by Jové et al. ${ }^{(32)}$ who report a $600 \%$ recovery of free $\mathrm{EC}$ in the caecal content of rats after providing them with a single dose of PA-rich

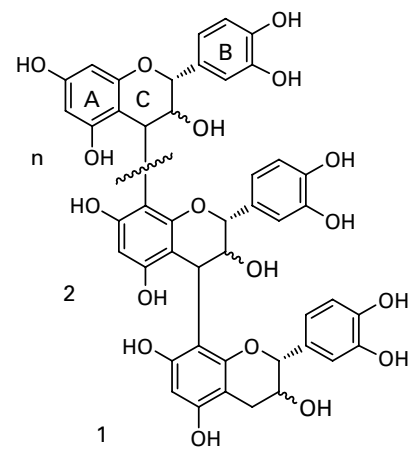
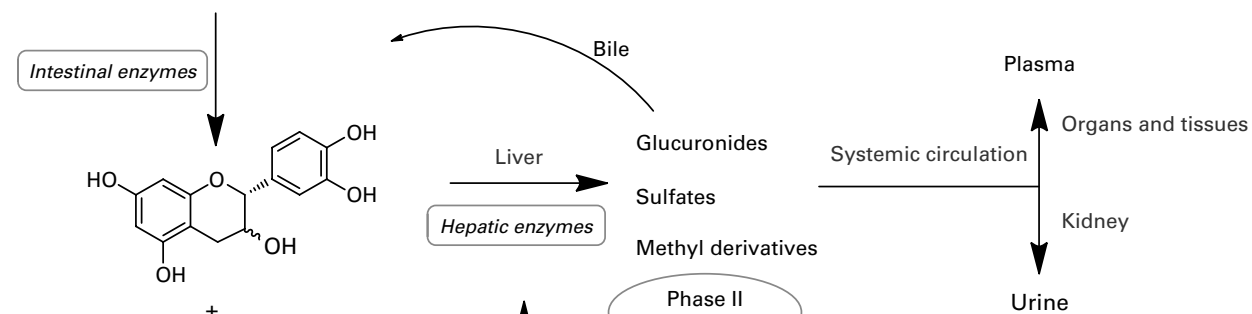

$\stackrel{+}{+}$

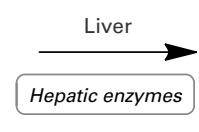

Glucuronides
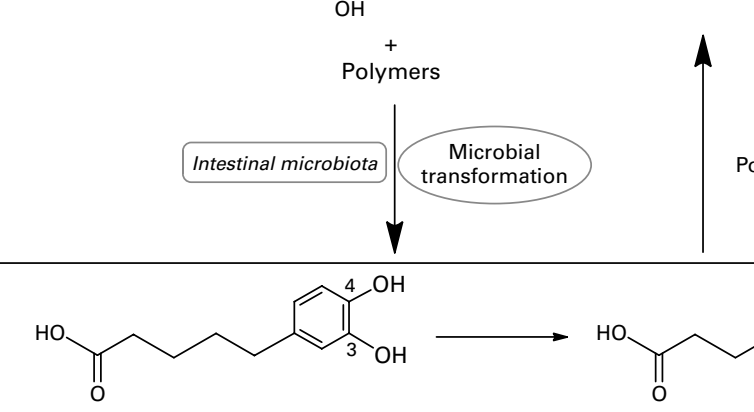<smiles>O=C(O)CCCCc1cccc(O)c1</smiles>

Microbial

metabolites

3,4-Dihydroxyphenylvaleric acid
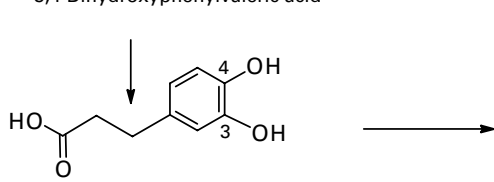

3,4-Dihydroxyphenylpropinoic acid

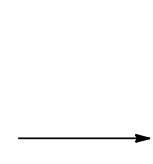

3-Hydroxyphenylvaleric acid

3-Hydroxyphenylpropinoic acid<smiles>O=C(O)Cc1cccc(O)c1</smiles>

3-Hydroxyphenylacetic acid

3,4-Dihydroxyphenylacetic acid<smiles>Cc1cc(O)c(O)cc1C(=O)O</smiles>

3,4-Dihydroxybenzoic acid
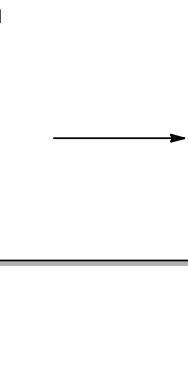

Faeces

Fig. 3. Metabolism of non-extractable proanthocyanidins (NEPA). 
almond extract. Moreover, the more than twenty EC-derived smaller metabolites detected in urine are consistent with the previous description of microbial fermentation and absorption. Our results also corroborate NEPA as PA, since direct evidence of the structure of these insoluble polymers is scarce. In fact, the residue after extraction with $70 \%$ acetone is commonly not considered to be a source of polyphenols. Our results agree with those which report that the residues of the common extraction with $70 \%$ acetone contain significant amounts of $\mathrm{PA}^{(12-14)}$.

The transformation of NEPA (Fig. 3) differs in part from the process suggested for the transformation of small EPA, previously described. In the case of NEPA, a proportion of the larger PA polymers appears to be depolymerised during their transit along the intestinal tract, resulting in delivery of EC monomers and possibly oligomers. The posterior degradation by the intestinal microbiota into small units may also differ between NEPA and EPA. As NEPA are associated with the food matrix in foodstuffs, particularly with other insoluble polymers constitutive of dietary fibre, their conversion may be slow compared to that of EPA. This deferred release would make NEPA metabolites bioavailable for particularly long times after intake and may result in them having health effects for a long time. This may explain the previously reported delay in the increase of plasma antioxidant capacity after the intake of GADF by human subjects compared to that observed after the intake of food rich in EPA, such as red wine ${ }^{(33)}$. In addition, the metabolites detected in faeces prove that putatively active species remain in contact with the colonic epithelium for at least $24 \mathrm{~h}$ after ingestion. Indeed, the intake of PA, and particularly PA with a high degree of polymerisation, has been associated with a reduced risk of colorectal cancer ${ }^{(7)}$ and our results suggest that food sources of NEPA could provide such putative cancerpreventative PA.

Previous nutritional studies have considered the extractable fraction of PA as the only source of dietary polyphenols. We show here that NEPA should be taken into account as most of the food in these studies contains significant amounts of NEPA. Further work is needed, both on the systematic analysis of NEPA in foodstuffs and on the metabolism of NEPA from different food sources, in order to unravel the contribution of this fraction of dietary PA to the health-promoting effects of fruit and vegetables.

In conclusion, we show here that NEPA are a source of polymeric PA that are progressively depolymerised during their transit along the intestinal tract into EC monomers and dimers, and later metabolised by the intestinal microbiota into smaller units. As a result, EC, phenolic acids and their phase II metabolites are in contact with the intestinal tract and bioavailable for at least $24 \mathrm{~h}$ after ingestion.

\section{Acknowledgements}

The present work was supported by the Spanish Ministry of Education and Science (AGL2009-12374-C03-03/ALI). J. P.-J. thanks the Spanish Ministry of Science and Innovation for granting her a Sara Borrell postdoctoral contract
(CD09/00068). GADF was a generous gift from Professor Fulgencio Saura-Calixto, ICTAN-CSIC. None of the authors had any conflict of interest. J. L. T., J. P.-J. and M. L. M.-M. designed the research. M. L. M.-M. and J. P.-J. carried out the experimental work. M. L. M.-M., J. P.-J. and E. F. analysed the data. M. L. M.-M. and J. P.-J. wrote the first version of the manuscript. All the authors contributed to writing the manuscript and approved the final version. Language revision by Christopher Evans is appreciated.

\section{References}

1. Serrano J, Puupponen-Pimiä R, Dauer A, et al. (2009) Tannins: current knowledge of food sources, intake, bioavailability and biological effects. Mol Nutr Food Res 53, S310-S329.

2. Zern TL, Wood RJ, Greene C, et al. (2005) Grape polyphenols exert a cardioprotective effect in pre- and postmenopausal women by lowering plasma lipids and reducing oxidative stress. J Nutr 135, 1911-1917.

3. Santos-Buelga C \& Scalbert A (2000) Proanthocyanidins and tannin-like compounds - nature, occurrence, dietary intake and effects on nutrition and health. J Sci Food Agric 80, $1094-1117$.

4. Bladé C, Arola L \& Salvadó MJ (2010) Hypolipidemic effects of proanthocyanidins and their underlying biochemical and molecular mechanisms. Mol Nutr Food Res 54, 37-59.

5. Montagut G, Bladé C, Blay M, et al. (2010) Effects of a grapeseed procyanidin extract (GSPE) on insulin resistance. J Nutr Biochem 21, 961-967.

6. Yokozawa T, Kim HJ \& Cho EJ (2008) Gravinol ameliorates high-fructose-induced metabolic syndrome through regulation of lipid metabolism and proinflammatory state in rats. J Agric Food Chem 56, 5026-5032.

7. Rossi M, Bosetti C, Negri E, et al. (2010) Flavonoids, proanthocyanidins and cancer risk: a network of casecontrol studies from Italy. Nutr Cancer 62, 871-877.

8. Gu L, Kelm MA, Hammerstone JF, et al. (2004) Concentrations of proanthocyanidins in common foods and estimations of normal consumption. J Nutr 134, 613-617.

9. Arranz S, Saura-Calixto F, Shaha S, et al. (2009) High contents of nonextractable polyphenols in fruits suggest that polyphenol contents of plant foods have been underestimated. J Agric Food Chem 57, 7298-7303.

10. Goñi I, Díaz-Rubio ME, Pérez-Jiménez J, et al. (2009) Towards an updated methodology for measurement of dietary fiber, including associated polyphenols, in food and beverages. Food Res Int 42, 840-846.

11. Report A (2001) The definition of dietary fibre. Cereal Foods World 46, 112-126.

12. Hellström JK \& Mattila PH (2008) HPLC determination of extractable and unextractable proanthocyanidins in plant materials. J Agric Food Chem 56, 7617-7624.

13. White BL, Howard LR \& Prior RL (2010) Release of bound procyanidins from cranberry pomace by alkaline hydrolysis. J Agric Food Chem 58, 7572-7579.

14. Pérez-Jiménez J, Arranz S \& Saura-Calixto F (2009) Proanthocyanidin content in foods is largely underestimated in the literature data: an approach to quantification of the missing proanthocyanidins. Food Res Int 42, 1381-1388.

15. Abia R \& Fry SC (2001) Degradation and metabolism of 14Clabelled proanthocyanidins from carob (Ceratonia siliqua) pods in the gastrointestinal tract of the rat. J Sci Food Agric 81, 1156-1165. 
16. Donovan JL, Manach C, Rios L, et al. (2002) Procyanidins are not bioavailable in rats fed a single meal containing a grapeseed extract or the procyanidin dimer B3. Br J Nutr 87, 299-306.

17. Tsang C, Auger C, Mullen W, et al. (2005) The absorption, metabolism and excretion of flavan-3-ols and procyanidins following the ingestion of a grape seed extract by rats. $\mathrm{BrJ}$ Nutr 94, 170-181.

18. Baba S, Osakabe N, Natsume M, et al. (2002) Absorption and urinary excretion of procyanidin B2 [epicatechin-(4[beta]-8)epicatechin] in rats. Free Radic Biol Med 33, 142-148.

19. Appeldoorn MM, Vincken J-P, Aura A-M, et al. (2009) Procyanidin dimers are metabolized by human microbiota with 2-(3,4-dihydroxyphenyl)acetic acid and 5-(3,4-dihydroxyphenyl)- $\boldsymbol{\gamma}$-valerolactone as the major metabolites. $J$ Agric Food Chem 57, 1084-1092.

20. Stoupi S, Williamson G, Drynan JW, et al. (2010) Procyanidin B2 catabolism by human fecal microflora: partial characterization of 'dimeric' intermediates. Arch Biochem Biophys 501, $73-78$.

21. Urpí-Sardá M, Garrido I, Monagas M, et al. (2009) Profile of plasma and urine metabolites after the intake of almond [Prunus dulcis (Mill.) D.A. Webb] polyphenols in humans. J Agric Food Chem 57, 10134-10142.

22. Neveu V, Pérez-Jiménez J, Vos F, et al. (2010) Phenolexplorer: an online comprehensive database on polyphenol contents in foods. Database, vol. 2010: article ID bap 024; doi:10.1093/database/bap024.

23. Rios LY, Gonthier M-P, Rémésy C, et al. (2003) Chocolate intake increases urinary excretion of polyphenol-derived phenolic acids in healthy human subjects. Am J Clin Nutr 77, 912-918

24. Touriño S, Fuguet E, Vinardell MP, et al. (2009) Phenolic metabolites of grape antioxidant dietary fiber in rat urine. J Agric Food Chem 57, 11418-11426.
25. Touriño S, Pérez-Jiménez J, Mateos-Martín ML, et al. (2011) Metabolites in contact with the rat digestive tract after ingestion of a phenolic-rich dietary fiber matrix. J Agric Food Chem 59, 5955-5963.

26. Saura-Calixto F, Pérez-Jiménez J, Touriño S, et al. (2010) Proanthocyanidin metabolites associated with dietary fibre from in vitro colonic fermentation and proanthocyanidin metabolites in human plasma. Mol Nutr Food Res 54, 939-946.

27. Pérez-Jiménez J, Serrano J, Tabernero M, et al. (2008) Effects of grape antioxidant dietary fiber in cardiovascular disease risk factors. Nutrition 24, 646-653.

28. Touriño S, Fuguet E, Jáuregui O, et al. (2008) Highresolution liquid chromatography/electrospray ionization time-of-flight mass spectrometry combined with liquid chromatography/electrospray ionization tandem mass spectrometry to identify polyphenols from grape antioxidant dietary fiber. Rapid Commun Mass Spectrom 22, 3489-3500.

29. Saura-Calixto FD \& Larraruri García JA (1999) Concentrate of natural antioxidant dietetic fiber from grape, and preparation process. CSIC, ES21300092 A1 A1.

30. Wishart DS, Tzur D, Knox C, et al. (2007) HMDB: the human metabolome database. Nucleic Acids Res 35, Database issue, D521-D526.

31. Das NP (1971) Studies on flavonoid metabolism: absorption and metabolism of $(+)$-catechin in man. Biochem Pharmacol 20, 3435-3445.

32. Jové M, Serrano JCE, Ortega N, et al. (2011) Multicompartmental LC-Q-TOF-based metabonomics as an exploratory tool to identify novel pathways affected by polyphenol-rich diets in mice. J Proteome Res 10, 3501-3512.

33. Pérez-Jiménez J, Serrano J, Tabernero M, et al. (2009) Bioavailability of phenolic antioxidants associated with dietary fiber: plasma antioxidant capacity after acute and longterm intake in humans. Plant Foods Hum Nutr 64, 102-107. 